\title{
An overview on Philippine estuarine oomycetes
}

\author{
Reuel M. Bennett ${ }^{1}$ and Marco Thines ${ }^{2,3}$
}

\begin{abstract}
Estuarine saprotrophic oomycetes are a group of eukaryotic, fungal-like protists of the Kingdom Straminipila. Species classified as estuarine oomycetes are commonly present on mangrove leaf litter and saltmarsh plant debris. They are distributed over several families (i.e. Peronosporaceae, Pythiaceae, Salisapiliaceae, and Salispinaceae). It is estimated that there are more than 100 species of estuarine oomycetes and, surprisingly, some supposedly terrestrial phytopathogenic hemibiotrophic oomycetes, e.g. Phytophthora elongata, Ph. insolita, and Ph. ramorum, are likewise present in the estuarine biome. In the Philippines, this group has been neglected for several decades as compared to the obligate biotrophic and hemibiotrophic members of Peronosporaceae and Albuginaceae. In this account, a general overview on the systematics and phylogeny of estuarine oomycetes is given. Further, the state of knowledge regarding thallus organization, taxonomy, habitat, and status of Philippine oomycetes are presented.
\end{abstract}

Keywords: estuarine, mangroves, oomycetes, phylogeny, taxonomy

\section{History of knowledge on estuarine oomycetes from saltmarsh and mangrove habitats}

The Phylum Oomycota is a group of fungal-like eukaryotes of the Kingdom Straminipila and is composed of approximately 1,700 species grouped into 90 genera (Beakes and Thines 2017, Wijayawardene et al. 2020). Studies conducted on oomycetes are focused mainly on the pathogenic groups, which include white blister rusts (e.g. Albugo spp., Pustula spp.), downy mildews (e.g. Bremia spp., Peronospora spp., Plasmopara spp.), Phytophthora spp., Pythium spp., and fish pathogens (e.g. Saprolegnia spp.). An ecologically important group of oomycetes that is hardly investigated are the saprotrophic oomycetes from saltmarsh, estuarine, or mangrove

\footnotetext{
${ }^{1}$ Department of Biological Sciences, College of Science, University of Santo Tomas, Manila, Philippines

${ }^{2}$ Senckenberg Biodiversity and Climate Research Centre, Senckenberg Nature Research, Georg-Voigt-Str. 14-16, 60325 Frankfurt am Main, Germany

${ }^{3}$ Department of Biological Sciences, Institute of Ecology and Evolution, Goethe University Frankfurt am Main, Max-von-Laue-Str. 9, 60438 Frankfurt am Main, Germany

*Corresponding email: rmbennett@ust.edu.ph

Date Submitted: 27 March 2020

Date Accepted: 02 June 2020
}

environments (Fig. 1). Nigrelli and Thines (2013) suggested that there are approximately 60 known species of marine oomycetes recorded in the literature, and to date, 30 species are known from mangrove and saltmarsh habitats (Hulvey et al. 2010, Nigrelli and Thines 2013, Bennett and Thines 2017, 2019, Bennett et al. 2017a, 2017b, 2017c, 2018).

Previously, an oomycete isolated from the rhizosphere, a leaf or any water sample having ovoid, obovoid, obpyriform, pyriform, ellipsoid or fusiform sporangia, and is cultivable, was usually considered a member of the genus Phytophthora (Bennett and Thines 2017, Bennett et al. 2017b). Phytophthora vesicula, a saprotrophic oomycete, was the first Phytophthora species reported from the marine environment (Anastasiou and Churchland 1969). This was then followed by Fell and Master (1975), Pegg and Alcorn (1982), and Gerrettson-Cornell and Simpson (1984) who reported additional mangrove oomycetes (Ph. avicenniae, Ph. bahamensis, Ph. batemanensis, Ph. epistomium, Ph. mycoparasitica, Ph. operculata, Ph. polymorphica, Ph. spinosa var. spinosa, and Ph. spinosa var. lobata). Ho and Jong (1990) proposed Halophytophthora as a generic concept for the Phytophthora-like species from the estuarine or mangrove ecosystem, to accommodate the abovementioned species. This resulted in their transfer to Halophytophthora, despite the broad generic circumscription in terms of morphology needed for this. Nonetheless, this concept was accepted for approximately two decades and resulted in the 

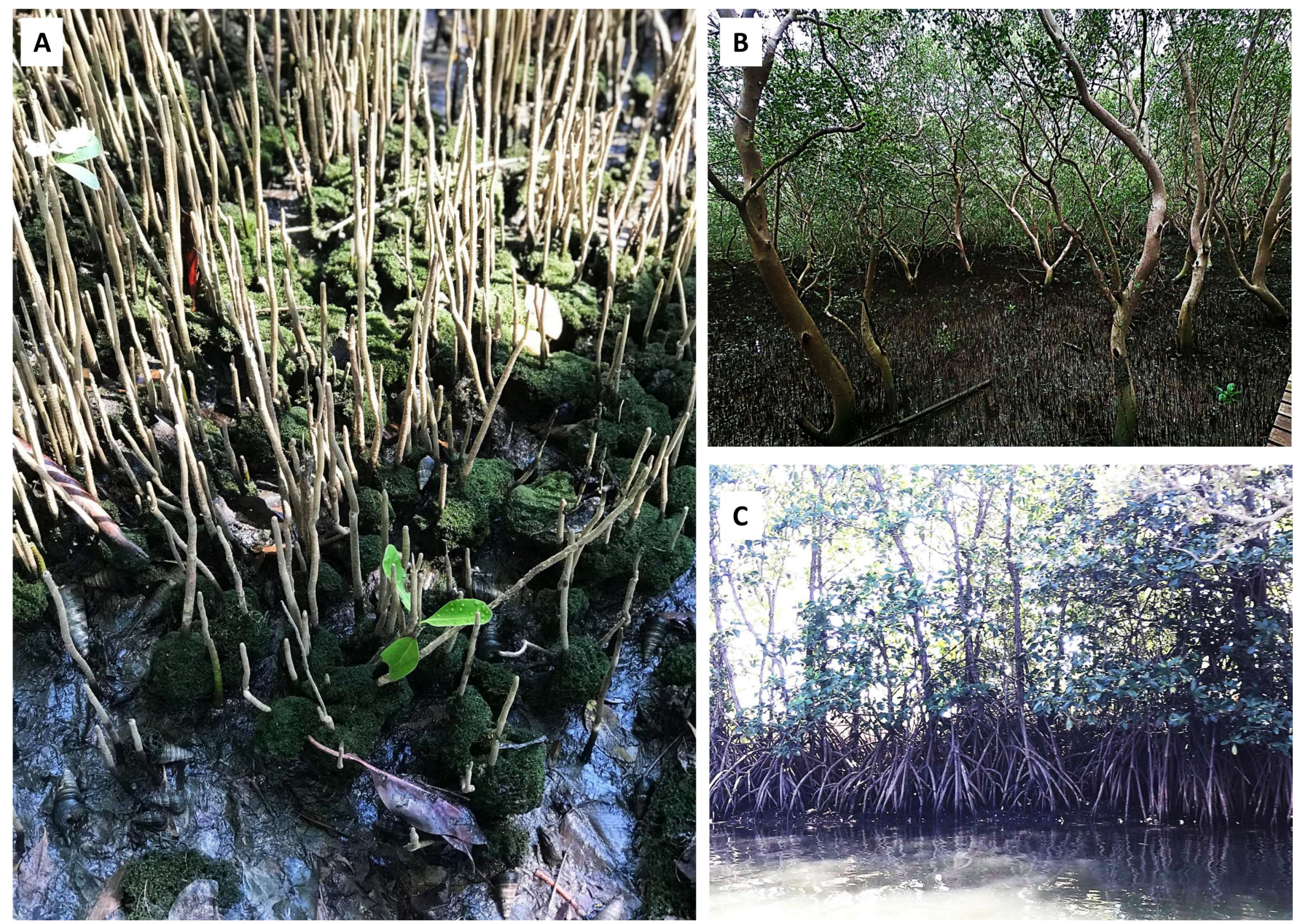

Figure 1. A mangrove sampling site in the Philippines (Image by Gabrielle Beatrix B. Francisco). Typical for mangroves is their distinct root system. (A-B) Pneumatophores. (C) Stilt root system. There are 40 known species of mangrove-forming plants in the Philippines, distributed over 16 families (Primavera 2000).

addition of several species, i.e. Halophytophthora elongata, $H$. exoprolifera, $H$. kandeliae, $H$. masteri, $H$. tartarea, and $H$. porrigovesica (Ho et al. 1991, Ho et al. 1992, Nakagiri et al. 1994, Nakagiri et al. 2001, Ho et al. 2003). However, a freshwater species, H. fluviatilis (Yang and Hong 2014), was isolated from a stream in the USA using Rhododendron as a bait, and, to date, is the only known freshwater congener. Nakagiri (2002) suggested that $H$. spinosa was not a member of Halophytophthora and was seen to be closely related to Sapromyces (Rhipidiaceae). This questioned the integrity of Halophytophthora as a monophyletic group and likewise with the generic concept of Phytophthora-like species from the estuarine or mangrove environment. In the studies of Huvley et al. (2010) and Lara and Belbahri (2011) the polyphyly of Halophytophthora was ascertained, setting the stage for further revision.
Hulvey et al. (2010) proposed the family Salisapiliaceae to accommodate species from the saltmarsh environment. This family is strongly supported phylogenetically and was monogeneric, comprising only Salisapilia. Three species were included in this genus, Salisapilia sapeloensis (type species), $S$. nakagirii, and $S$. tartarea (basionym: Halophytophthora tartarea). The genus Phytopythium (Bala et al. 2010) was proposed to accommodate species having shared characteristics of Pythium and Phytophthora. This monophyletic group was previously referred to as the clade $\mathrm{K}$ based in the phylogenetic study of the genus Pythium (Lévesque and de Cock 2004). However, Halophytophthora kandeliae is nested in this genus, and as a result, Thines (2014) proposed the combination Phytopythium kandeliae, which was supported by strong morphological and phylogenetic evidence. More recently, Salispina was proposed to accommodate the two varieties of $H$. spinosa in Salispina as S. spinosa and S. lobata together with the 
type species, S. intermedia (Li et al. 2016). However, Salispina was placed as the sister taxon to Sapromyces rather than Peronosporaceae or Salisapiliaceae (Li et al. 2016). But due to low support for this seemingly unusual grouping, no higher level placement of the genus Salispina has been proposed.

\section{Overview of the estuarine oomycetes phylogeny}

Currently, the cultivable estuarine oomycetes are classified into three families, Peronosporaceae, Salispinaceae, and Salisapiliaceae (Fig. 2). The family Peronosporaceae contains complex assemblages of oomycete species including the large group of downy mildews (e.g. Bremia, Peronospora, Plasmopara), Calycofera, Phytophthora, Halophytophthora, and Phytopythium (Bennett et al. 2017a, 2017c, Beakes and Thines 2017).

Apart from downy mildew genera, Phytopythium and Calycofera are the only monophyletic taxa, whereas the others are either paraphyletic (i.e. Phytophthora) or still polyphyletic (i.e. Halophytophthora) (Bennett et al. 2018), despite the recent classification of several species of Halophytophthora within Salisapilia (Bennett and Thines 2019), as some are still not resolved in terms of their phylogenetic placement (e.g. $H$. exoprolifera and $H$. porrigovesica). Salisapiliaceae is a well- established taxon based on the phylogeny of Hulvey et al. (2010) and is composed of three species, Salisapilia sapeloensis (type species), S. nakagirii, and S. tartarea. According to the recent revision of Bennett and Thines (2019), Salisapiliaceae now contains several additional species transferred from other genera (Salisapilia bahamensis, S. elongata, S. epistomia, S, masteri, and S. mycoparasitica) and a new species (S. coffeyi).

In line with its phylogenetic placement, Salispinaceae has recently been described as a taxon within Rhipidiales and is assumed to grow in an environment with reduced oxygen concentration (Bennett et al. 2018). Salispina is monogeneric and members are either partly or entirely aculeolate. Members of Salispina are reported as estuarine species, whereas Rhipidiaceae thrive in freshwater biomes (Dick 2001). In the Philippines, Salispina hoi, S. lobata, and S. spinosa have been isolated from mangrove leaf litter (Leaño 2001, Bennett et al. 2018, Caguimbal et al. 2019).

\section{Sporangia and gametangia: Structures for survival and perpetuation}

Oomycetes thrive in different moist biomes due to their ability to colonize substrates by actively swimming towards them, which results in their evolutionary success (Thines 2014).
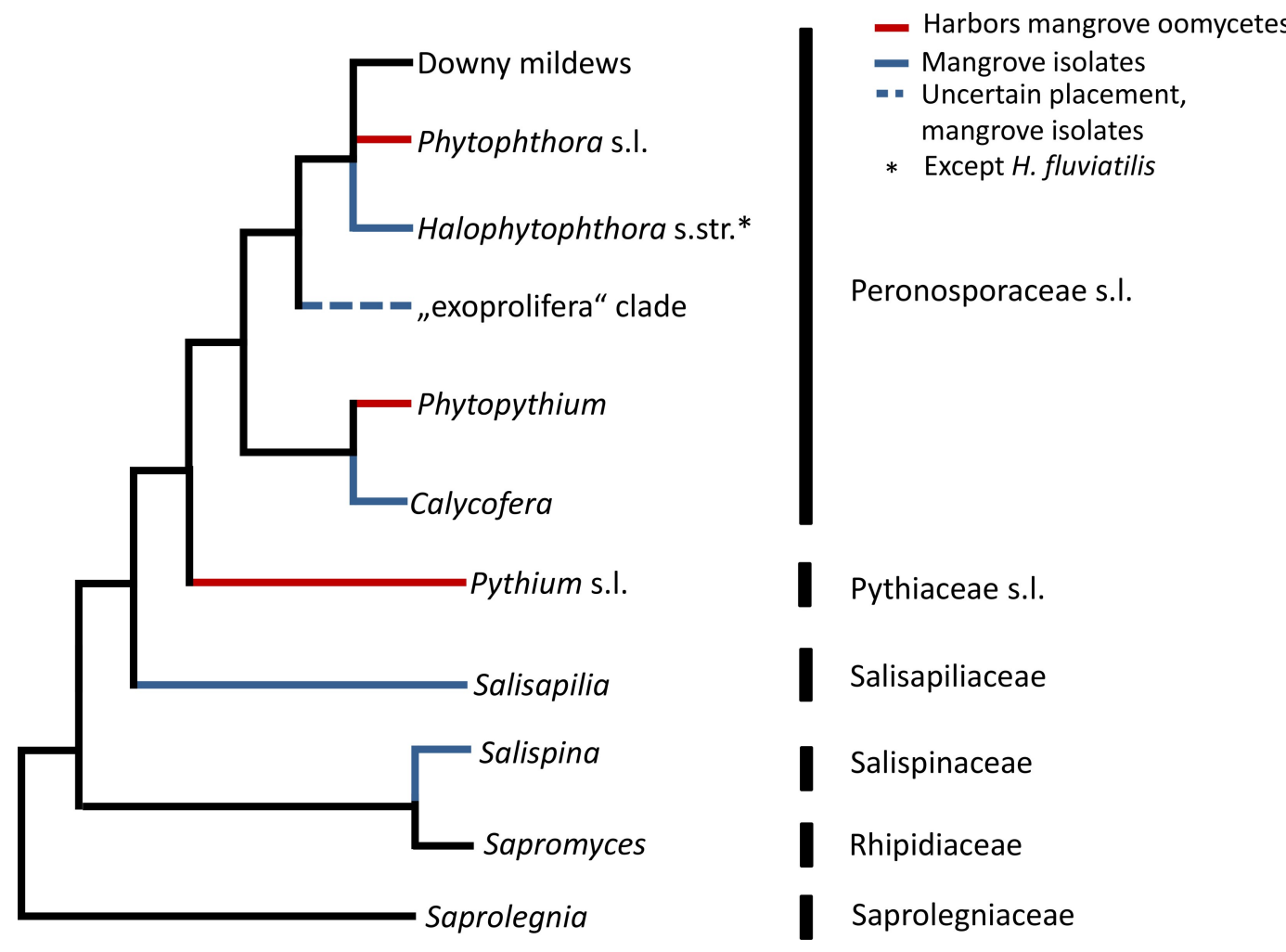

Figure 2. An overview of the phylogeny of estuarine and mangrove oomycetes (after Hulvey et al. 2010, Nigrelli and Thines 2013, Thines 2014, Li et al. 2016, Bennett et al. 2018, Bennett and Thines 2019). 
Reproductive structures of saprotrophic oomycetes play a role in both colonization and survival (Fig. 3). Sporangia (or zoosporangia), which are formed through sporulation, are pouch -like structures of different shapes in which zoospores are formed (Figs. 3a - e). Zoospores are diploid, biflagellate, heterokont cells that are chemotactically attracted to different plant exudates (Leaño et al. 2000). They are formed after mitotic division of the protoplasmic component of sporangia. After complete maturity of zoospores, as triggered by external stimuli, they are released from the sporangia. Once zoospores locate a suitable substrate for colonization, they retract their flagella, encyst, and germinate, forming a germ-tube that will later develop into a dense mycelium (Leaño et al. 2000).

Oomycetes develop oogonia (female) and antheridia (male), which are collectively known as gametangia (Figs. $3 \mathrm{f}-$ i). Antheridium attachment is a taxonomically informative feature in several genera and is described as either paragynous when the antheridium is on the side of the oogonium or amphigynous when the antheridium surrounds the base of the oogonium. Some species of saprotrophic or hemibiotrophic oomycetes have constricted to roundish antheridia (Bala et al. 2010, de Cock et al. 2015) (Figs. 3f - h). The origin of antheridia from vegetative hyphae is a taxonomically valuable character in several groups and is classified as either monoclinous when the antheridium branches from the hypha bearing the oogonium or diclinous when the antheridial hypha is separate from the hypha bearing the oogonium. Projections on the oogonial wall are somehow varied among and within the groups and can be highly ornamented in Pythium spp. (van der Plaats-Niterink 1981). An oospore forms after fertilization of the oosphere by a nucleus transferred from the antheridium. Oospores are thick-walled resting zygotes and are capable of long-term survival in soil, water, or plant tissue. Once environmental conditions favor germination, the oospore develops a germ tube, and either a new mycelial network is formed or primary zoosporangium is released. In heterothallic species, where antheridia and oogonia are formed by distinct mycelia, the presence of both mating types is necessary for oospore formation, whereas in homothallic species sexual reproduction mostly takes place by selfing.

\section{Ecology of mangrove oomycetes}

Ecologically, Halophytophthora are ubiquitous decomposers living in mangrove leaf litter (Nakagiri 2000,
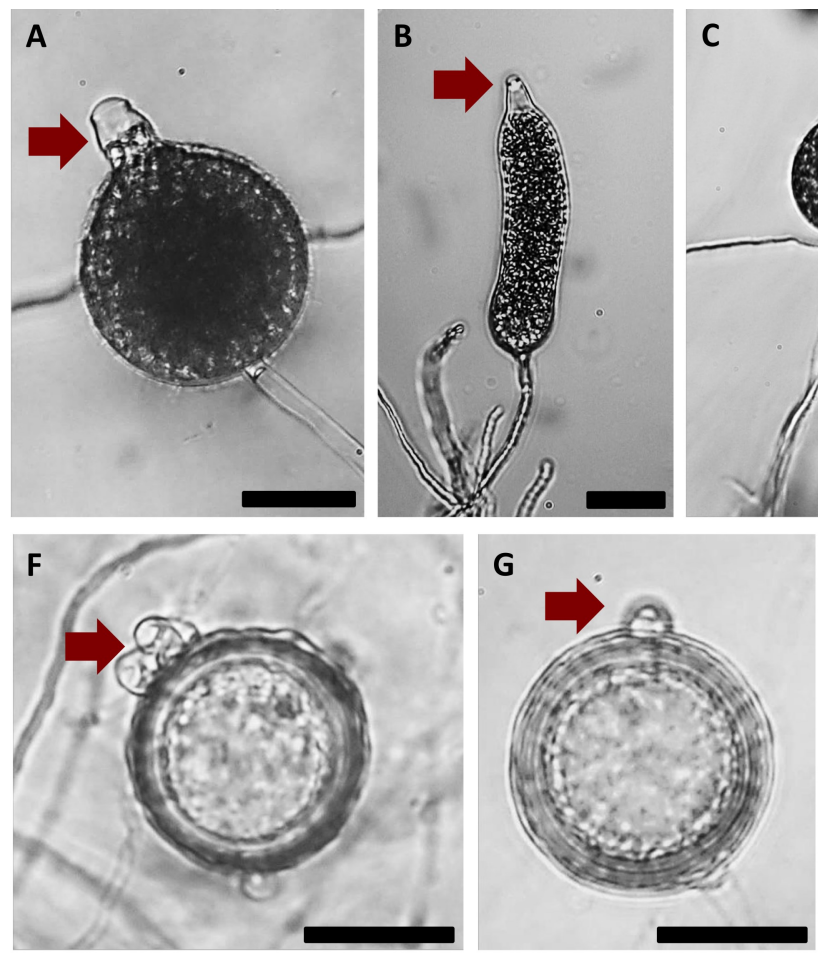
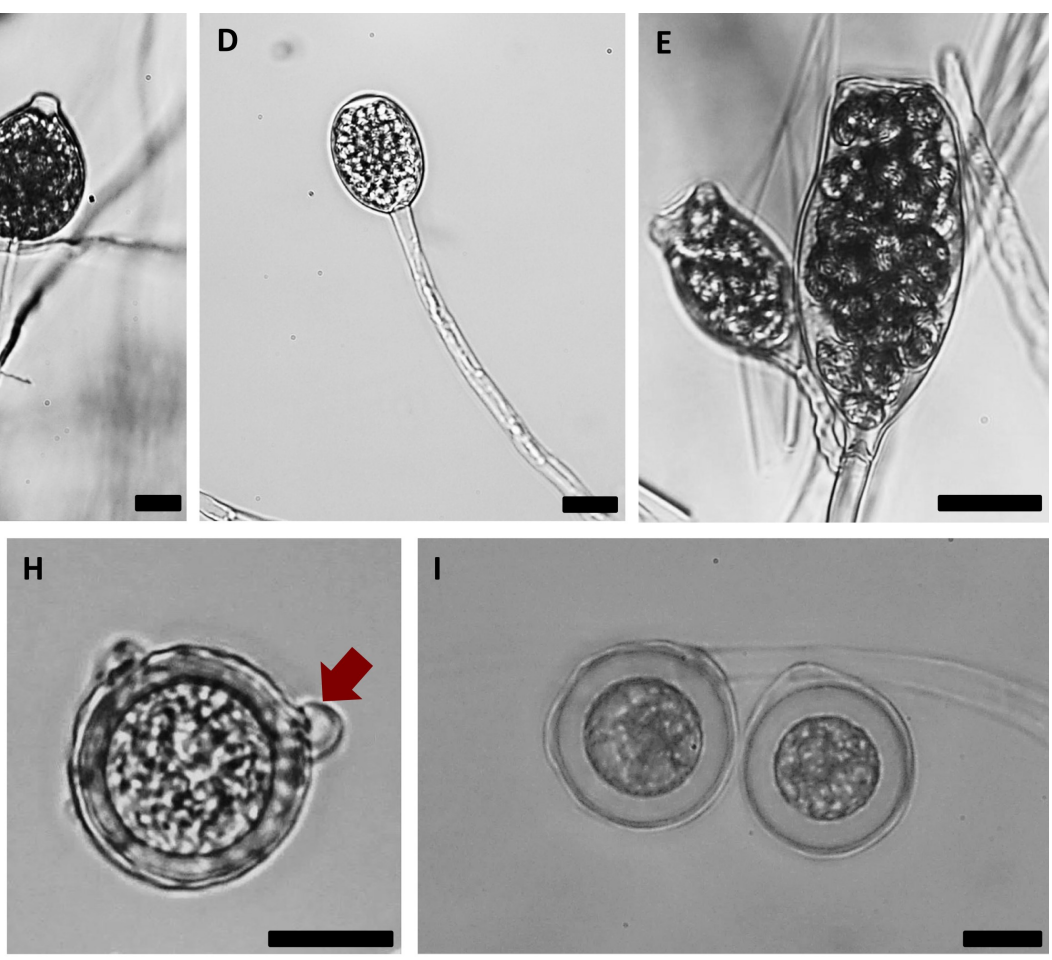

Figure 3. Sporangia (A-E) of A Salisapilia nakagirii (Bennett and Thines 2019) (arrow points at the discharge tube). B Salisapilia elongata (Bennett and Thines 2019) (arrow points at the discharge tube with the typical hyaline apical plug). C Halophytophthora batemanensis. D Halophytophthora porrigovesica. and E Calycofera operculata (Bennett et al. 2017a) . F-I Gametangia $\mathbf{F}-\mathbf{H}$ of Phytopythium leanoi (Bennett et al. 2017c) (arrow points at the antheridium), and I Phytophthora insolita (Bennett et al. 2017). Scale bars $=20 \mu \mathrm{m}$. 
Leaño 2001), driftwoods (Leong et al. 1998; Tan and Leong 1992), marsh grass litter (Hulvey et al. 2010), and in Avicennia marina - trunk cankers and decayed rootlets (Pegg and Foresberg 1981). In Asian countries, mangrove leaves are common habitats of oomycetes and can be colonized from the initial to the final stage of leaf decay (Leaño et al. 2000). Of the different mangrove plant genera, Halophytophthora spp. were reported to colonize Avicennia, Aegiceras, Bruguiera, Ceriops, Rhizophora, Sonneratia, and Xylocarpus (Leaño 2001, Nakagiri 2000, Pegg and Forsberg 1981, Tan and Pek 1997). Associations between mangrove species and oomycetes observed so far are saprotrophic, and in the current literature no study has reported oomycetes as pathogens of mangrove plants. However, Halophytophthora mycoparasitica has been reported as mycoparasite of fungi such as Pestalotia sp. and Penicillium sp. (Fell and Master 1975).

Halophytophthora spp. are considered to be the first colonizers of fallen mangrove leaves (Newell et al. 1987, Nakagiri et al. 1989). These species play an important role as decomposers. Once a senescent mangrove leaf falls into the water system, zoospores of Halophytophthora spp. are chemotactically attracted to the leaf leachates and attach rapidly onto the surface of the leaf and colonize it via mycelial extension, and several enzymes (e.g. cellulase) are produced to degrade the leaves (Raghukumar et al. 1994). Decomposition of leaf substrates occurs rapidly due to the quick infestation by zoospores (Nakagiri et al. 1989). The decomposition process mediated by Halophytophthora spp. and other saprotrophic oomycetes also transforms the leaves into an accessible food source for small invertebrates (Fell and Master 1980).

Responses of mangrove oomycetes to abiotic factors (e.g. temperature, salinity, and $\mathrm{pH}$ ) were comprehensively summarized by Leaño et al. (2000). They inferred that Halophytophthora spp. are well-adapted to a wide range of $\mathrm{pH}$ $(6.0-9.0)$, salinity $(0-60 \%)$ and temperature $\left(10-35^{\circ} \mathrm{C}\right)$ and some were able to grow and sporulate throughout these ranges. These ranges correspond to the constant fluctuation of abiotic factors in a mangrove ecosystem and is a probable reason supporting their abundance in such habitats (Leaño et al. 2000).

In line with this, using $H$. vesicula as a model organism, Leaño (2001) attributed the ecological success of Halophytophthora spp. in colonizing mangrove leaves to their abundant zoospore production, wide tolerance to $\mathrm{pH}$, salinity, and temperature, ability to compete against higher fungi in colonizing mangrove leaves, and ability of zoospores to quickly attach firmly to a substrate.

\section{Oomycetes in the Philippines}

Oomycete research in the Philippines is relatively young as until recently, only a few species have been recorded in the scientific literature dealing with Philippine ecosystems. In the end of the 1980s, genera had been reported from the soil, and freshwater and marine environments as saprotrophs (e.g. Halophytophthora spp.) or pathogens (e.g. Phytophthora spp., Pythium spp., Haliphthoros spp., downy mildews and white blister rust), but these reports remained mostly anecdotal. After the 1980s, studies on Philippine oomycetes became stagnant with only a few studies carried compared to neighboring Asian countries (e.g. China, Japan, Taiwan, Thailand).

Increased interest in Halophytophthora species started in the 1990s, however, studies on the systematics, distribution, and ecology of Halophytophthora in the Philippines are very limited. Leaño (2001) reported the occurrence of Halophytophthora in Panay, listing five species namely $H$. vesicula, Phytopythium kandeliae ( $\equiv H$. kandeliae), Salisapilia bahamensis ( $\equiv P h$. bahamensis, H. bahamensis), S. epistomium (三Ph. epistomium, H. epistomium), and Salispina lobata $(\equiv P h$. spinosa var. lobata, H. spinosa var. lobata), and one unidentified Halophytophthora species. A decade later this was followed by Antazo et al. (2012) who isolated three unidentified Halophytophthora spp. from Marinduque. The number of mangrove oomycete species for the Philippines was updated by Bennett and Thines (2017), Bennett et al. (2017a, 2017b, 2017c, 2018), and Bennett and Thines (2019). Phytopythium dogmae (epithet named after Irineo Dogma Jr.), Phytopythium leanoi (named after Eduardo Leaño), and Salispina hoi (named after Hon Ho) are recently described taxa isolated from the Philippines. In addition, several species were newly reported for the Philippines, e.g. Ph. insolita, Ph. elongata, and S. bahamensis. Recently, several studies explored the fatty acid composition of Philippine mangrove oomycetes, and some isolates produced good amounts of polyunsaturated fatty acids (Say et al. 2017, Caguimbal et al. 2019). Despite these taxonomic proposals and applied studies, oomycete research in the Philippines, particularly reagarding the saprotrophic and marine groups, is still underexplored.

From the limited number of species known in relation to the geographical position, climate and ecological diversity of the Philippines, it can be assumed that Halophytophthora and other marine oomycetes (e.g. Phytopythium, Salisapilia) are abundant and diverse, with most of the species awaiting discovery. Table 1 is a non-exclusive list of oomycetes recorded in the Philippines so far.

\section{Current status and future directions}

Of the approximately 1,700 species of known oomycetes (Thines 2014, Wijayawardene et al. 2020), 30 species (approx. $1.5 \%)$ are considered as mangrove or saltmarsh oomycetes (Table 2). Species classified in this group are distributed in three 
Table 1. Philippine oomycetes recorded in the literature

\begin{tabular}{|c|c|c|c|}
\hline Species & Isolation source / host or substrate & Family* & References \\
\hline Lagenidium oophilum & Parasite, nematode eggs & & \\
\hline \multirow[t]{2}{*}{ Myzocytium megastomum } & $\begin{array}{l}\text { Obligate endoparasite, Cladophora, } \\
\text { Pithophora }\end{array}$ & & Dogma 1986 \\
\hline & Closterium & & Dogma 1975 \\
\hline Myzocytium proliferum & Spirogyra & & Dogma 1975 \\
\hline Albugo candida & Obligate pathogen, crucifers & Albuginaceae & Dogma 1986 \\
\hline Albugo ipomoeae-panduratae & Obligate pathogen, Ipomoea & Albuginaceae & Dogma 1986 \\
\hline Haliphthoros milfordensis & Pathogen, Scylla serrata & Haliphthoraceae & Leaño 2002 \\
\hline Haliphthoros philippinensis & $\begin{array}{l}\text { Pathogen, Penaeus monodon } \\
\text { Scylla serrata }\end{array}$ & Haliphthoraceae & $\begin{array}{l}\text { Hatai et al. } 1980 \\
\text { Leaño } 2002\end{array}$ \\
\hline Lagenidium giganteum & Saprotroph, parasite of mosquito larvae & Lagenidiaceae & Dogma 1986 \\
\hline Lagenidium humanum & Saprotroph, soil & Lagenidiaceae & Dogma 1986 \\
\hline Lagenidium pygmaeum & Saprotroph, soil and freshwater & Lagenidiaceae & Dogma 1986 \\
\hline Leptolegniella keratinophilum & $\begin{array}{l}\text { Soil } \\
\text { (baited using snake skin, hair) }\end{array}$ & Leptolegniellaceae & Dogma 1986 \\
\hline Apodachlya minima & Soil, saprotroph & Leptomitaceae & Dogma 1986 \\
\hline Leptomitus lacteus & Saprotroph, foul and polluted waters & Leptomitaceae & Dogma 1986 \\
\hline Olpidiopsis karlingae & Parasite, Karlingia rosea & Olpidiopsidaceae & Dogma 1986 \\
\hline Olpidiopsis luxurians & Parasite, Aphanomyces laevis & Olpidiopsidaceae & Dogma 1986 \\
\hline Olpidiopsis pythii & Parasite, Pythium intermedium & Olpidiopsidaceae & Dogma 1975 \\
\hline Bremia lactucae & Obligate pathogen, lettuce & Peronosporaceae & Dogma 1986 \\
\hline $\begin{array}{l}\text { Halophytophthora vesicula } \\
\text { (三Phytophthora vesicula) }\end{array}$ & $\begin{array}{l}\text { Saprotroph, Avicennia lanata, A. officinalis, } \\
\text { Ceriops decandra, } R \text {. apiculata, Sonneratia } \\
\text { sp., Xylocarpus granatum, X. moluccensis }\end{array}$ & Peronosporaceae & $\begin{array}{l}\text { Leaño } 2001 \\
\text { Caguimbal et al. } 2019\end{array}$ \\
\hline $\begin{array}{l}\text { Peronosclerospora philippinensis } \\
\text { (三Sclerophthora philippinensis) }\end{array}$ & $\begin{array}{l}\text { Obligate pathogen, corn } \\
\text { Saccharum, Sorghum, Euchlaena }\end{array}$ & Peronosporaceae & $\begin{array}{l}\text { Weston } 1920 \\
\text { Dogma } 1986\end{array}$ \\
\hline $\begin{array}{l}\text { Peronosclerospora sacchari } \\
\text { (三 Sclerophthora sacchari) }\end{array}$ & $\begin{array}{l}\text { Obligate pathogen, sugarcane, Euchlaena, } \\
\text { Tripsacum, Sorghum }\end{array}$ & Peronosporaceae & Dogma 1986 \\
\hline $\begin{array}{l}\text { Peronosclerospora sorghi } \\
(\equiv \text { Sclerophthora sorghi) }\end{array}$ & $\begin{array}{l}\text { Obligate pathogen, sorghum, Euchlaena, } \\
\text { Heteropogon, Panicum, Pennisetum, } \\
\text { Saccharum, Dichantium, Panicum }\end{array}$ & Peronosporaceae & Dogma 1986 \\
\hline $\begin{array}{l}\text { Peronosclerospora spontanea }(\equiv \\
\text { Sclerophthora spontanea) }\end{array}$ & Obligate pathogen, corn, Saccharum & Peronosporaceae & Dogma 1986 \\
\hline $\begin{array}{l}\text { Peronosclerospora miscanthi } \\
(\equiv \text { Sclerophthora miscanthi) }\end{array}$ & $\begin{array}{l}\text { Obligate pathogen, Miscanthus, Saccharum, } \\
\text { Sorghum, corn }\end{array}$ & Peronosporaceae & Dogma 1986 \\
\hline Phytophthora cactorum & Pathogen, Theobroma cacao & Peronosporaceae & $\begin{array}{l}\text { Mendiola and Espino } \\
1916\end{array}$ \\
\hline
\end{tabular}


Table 1 (cont.). Philippine oomycetes recorded in the literature

\begin{tabular}{|c|c|c|c|}
\hline Species & Isolation source / host or substrate & Family* & References \\
\hline Phytophthora capsici & Pathogen, Piper nigrum & Peronosporaceae & Tsao et al. 1994 \\
\hline Phytophthora citrophthora & $\begin{array}{l}\text { Pathogen, Citrus } \\
\text { Nephelium, Sandoricum }\end{array}$ & Peronosporaceae & $\begin{array}{l}\text { Del Rosario } 1968 \text {, } \\
\text { Tsao et al. } 1994\end{array}$ \\
\hline Phytophthora colocassiae & Pathogen, Colocasia esculenta & Peronosporaceae & $\begin{array}{l}\text { Mendiola and Espino } \\
1916\end{array}$ \\
\hline Phytophthora elongata & Decaying mangrove leaves & Peronosporaceae & Bennett et al. 2017b \\
\hline Phytophthora heveae & Pathogen, Sandoricum koetjape & Peronosporaceae & Tsao et al. 1994 \\
\hline Phytophthora infestans & $\begin{array}{l}\text { Pathogen, Solanum tuberosum } \\
\text { tomato, potato }\end{array}$ & Peronosporaceae & $\begin{array}{l}\text { Lee } 1921 \\
\text { Dogma } 1986\end{array}$ \\
\hline Phytophthora insolita & Decaying mangrove leaves & Peronosporaceae & Bennett and Thines 2017 \\
\hline Phytophthora meadii & Pathogen, Hevea brasiliensis & Peronosporaceae & Teodoro 1926 \\
\hline Phytophthora nicotianae & $\begin{array}{l}\text { Pathogen, Piper nigrum } \\
\text { Citrus } \\
\text { Solanum melongena } \\
\text { Carica papaya } \\
\text { Ananas comosus } \\
\text { Citrullus lanatus }\end{array}$ & Peronosporaceae & $\begin{array}{l}\text { Tsao et al. } 1994 \\
\text { Lee } 1921 \\
\text { Ocfemia } 1925 \\
\text { Quimio and Quimio } 1974 \\
\text { Quebral et al. } 1962 \\
\text { Quimio and Quimio } 1974\end{array}$ \\
\hline Phytophthora palmivora & Pathogen, omnivorous & Peronosporaceae & $\begin{array}{l}\text { Reinking 1919, Teodoro } \\
\text { 1926, Celino 1933 } \\
\text { Ela 1968, Del Rosario } \\
1968 \\
\text { Dogma 1986, Tsao et al. } \\
\text { 1994, Borines et al. } 2014\end{array}$ \\
\hline $\begin{array}{l}\text { Phytophthora parasitica } \\
\text { (synonym of } P \text {. nicotianae) }\end{array}$ & $\begin{array}{l}\text { Pathogen, Cocos, Theobroma, Gossypium, } \\
\text { Lycopersicum, Solanum, Hibiscus, Musa, } \\
\text { Citrus, Rheum, Lilium, Capsicum, Ananas, } \\
\text { Nicotiana, Ricinus, Grammatophyllum, Pollia, } \\
\text { Catharanthus, Manihot, Vigna, Bryophyllum }\end{array}$ & Peronosporaceae & Dogma 1986 \\
\hline Phytophthora phaseoli & Pathogen, Sandoricum koetjape & Peronosporaceae & Clara 1928 \\
\hline Phytopythium dogmae & Decaying mangrove leaves & Peronosporaceae & Bennett et al. 2017c \\
\hline $\begin{array}{l}\text { Phytopythium kandeliae } \\
\text { (三Halophytophthora kandeliae) }\end{array}$ & $\begin{array}{l}\text { Saprotroph, Avicennia lanata, } \\
\text { Rhizophora apiculata, Sonneratia sp. }\end{array}$ & Peronosporaceae & Leaño 2001 \\
\hline Phytopythium leanoi & Decaying mangrove leaves & Peronosporaceae & Bennett et al. 2017c \\
\hline Hyaloperonospora parasitica & Obligate pathogen, crucifers & Peronosporaceae & Dogma 1986 \\
\hline Plasmopara viticola & Obligate pathogen, grapes & Peronosporaceae & Dogma 1986 \\
\hline Pseudoperonospora cubensis & Obligate pathogen, cucurbits & Peronosporaceae & Dogma 1986 \\
\hline
\end{tabular}


Table 1 (cont.). Philippine oomycetes recorded in the literature

\begin{tabular}{|c|c|c|c|}
\hline Species & Isolation source / host or substrate & Family* & References \\
\hline Pythium aphanidermatum & $\begin{array}{l}\text { Pathogen, sugarcane, corn, sorghum, radish, } \\
\text { tobacco, Carica, cucumber, Solanum }\end{array}$ & Pythiaceae & Dogma 1986 \\
\hline Pythium arrhenomanes & Pathogen, corn, wheat, sugarcane & Pythiaceae & Dogma 1986 \\
\hline Pythium debaryanum & Pathogen, omnivorous species & Pythiaceae & Dogma 1986 \\
\hline Pythium echinulatum & $\begin{array}{l}\text { Saprotroph, normally can be pathogenic } \\
\text { similar to Py. debaryanum, Py. arrhenomanes, } \\
\text { and } P y \text {. aphanidermatum }\end{array}$ & Pythiaceae & Dogma 1986 \\
\hline Pythium monospermum & Saprotroph, soil and freshwater & Pythiaceae & Dogma 1986 \\
\hline Pythium proliferum & Saprotroph, or pathogen of strawberry & Pythiaceae & Dogma 1986 \\
\hline Pythium torulosum & Saprotroph, freshwater and very moist soils & Pythiaceae & Dogma 1986 \\
\hline $\begin{array}{l}\text { Salisapilia bahamensis } \\
(\equiv \text { Phytophthora bahamensis) }\end{array}$ & Saprotroph, Avicennia lanata & Salisapiliaceae & Leaño 2001 \\
\hline $\begin{array}{l}\text { Salisapilia elongata } \\
(\equiv \text { Halophytophthora elongata) }\end{array}$ & Decaying mangrove leaves & Salisapiliaceae & Bennett and Thines 2019 \\
\hline $\begin{array}{l}\text { Salisapilia epistomium } \\
\text { (三 Phytophthora epistomium) }\end{array}$ & $\begin{array}{l}\text { Saprotroph, Rhizophora apiculata, Sonneratia } \\
\text { sp., Xylocarpus granatum }\end{array}$ & Salisapiliaceae & Leaño 2001 \\
\hline Salispina hoi & Decaying mangrove leaves & Salispinaceae & Bennett et al. 2018 \\
\hline $\begin{array}{l}\text { Salispina lobata } \\
(\equiv \text { Phytophthora spinosa var. lobata) }\end{array}$ & Saprotroph, Xylocarpus moluccensis & Salispinaceae & Leaño 2001 \\
\hline $\begin{array}{l}\text { Salispina spinosa } \\
(\equiv \text { Phytophthora spinosa var. spinosa) }\end{array}$ & Decaying mangrove leaves & Salispinaceae & Caguimbal et al. 2019 \\
\hline Achlya ambisexualis & Freshwater, saprotroph & Saprolegniaceae & Dogma 1986 \\
\hline Achlya proliferoides & Freshwater, saprotroph & Saprolegniaceae & Dogma 1986 \\
\hline Achyla americana & Pathogen, Rice grain rot & Saprolegniaceae & Dogma 1986 \\
\hline Achyla bisexualis & Freshwater and moist soils & Saprolegniaceae & Dogma 1986 \\
\hline Achyla flagellata & Pathogen, Rice grain rot & Saprolegniaceae & Dogma 1986 \\
\hline Dictyuchus anomalus & Freshwater, soil, saprotroph & Saprolegniaceae & Dogma 1986 \\
\hline Saprolegnia diclina & $\begin{array}{l}\text { Pathogen of fish, apparently rare in the } \\
\text { Philippines }\end{array}$ & Saprolegniaceae & Dogma 1986 \\
\hline Thraustotheca clavata & Saprotroph, highland soils & Saprolegniaceae & Dogma 1986 \\
\hline Aphanomyces cladogamus & $\begin{array}{l}\text { Parasite, tomato, spinach, eggplant, lettuce, } \\
\text { sugar beet }\end{array}$ & Verrucalvaceae & Dogma 1986 \\
\hline Aphanomyces helicoides & Freshwater, saprotroph & Verrucalvaceae & Dogma 1986 \\
\hline Aphanomyces keratinophilus & Freshwater, saprotroph & Verrucalvaceae & Dogma 1986 \\
\hline Aphanomyces laevis & $\begin{array}{l}\text { Freshwater, saprotroph; or as parasite of some } \\
\text { desmids, crayfish, and Philippine milkfish } \\
\text { (Chanos sp.), catfish, carps }\end{array}$ & Verrucalvaceae & Dogma 1986 \\
\hline Plectospira gemmifera & Pathogen, sugarcane & Verrucalvaceae & Dogma 1986 \\
\hline
\end{tabular}

\footnotetext{
*based on Beakes and Thines 2017; Entries highlighted with grey are species reported in the mangrove environment.
} 
Table 2. Summary of mangrove or saltmarsh oomycetes

\begin{tabular}{|c|c|c|c|}
\hline & Substrate & Country & Reference \\
\hline \multicolumn{4}{|l|}{ Peronosporaceae } \\
\hline \multicolumn{4}{|l|}{ Phytophthora } \\
\hline Ph. elongata & Mangrove leaf litter & Australia, Philippines & Bennett et al. 2017a \\
\hline Ph. estuarina & $\begin{array}{l}\text { Laguncularia racemosa } \\
\text { Rhizophora mangle }\end{array}$ & Brazil & Li et al. 2016 \\
\hline Ph. insolita & Mangrove leaf litter & $\begin{array}{l}\text { India, South China, Philippines, } \\
\text { Taiwan, USA }\end{array}$ & Bennett and Thines 2017 \\
\hline Ph. inundata & Zostera marina & The Netherlands & Man In 'T Veld et al. 2011 \\
\hline Ph. gemini & Zostera marina & The Netherlands & Man In 'T Veld et al. 2011 \\
\hline Ph. rhizophorae & Rhizophora mangle & Brazil & Li et al. 2016 \\
\hline \multicolumn{4}{|l|}{ Halophytophthora } \\
\hline H. avicenniae & Avicennia marina & Australia, Taiwan & $\begin{array}{l}\text { Gerrettson-Cornell and } \\
\text { Simpson 1984, Pang et al. } \\
2014\end{array}$ \\
\hline H. batemanensis & $\begin{array}{l}\text { Mangrove leaf litter } \\
\text { Avicennia marina }\end{array}$ & Australia, Japan, Philippines & $\begin{array}{l}\text { Gerrettson-Cornell and } \\
\text { Simpson 1984, Nakagiri } 2000\end{array}$ \\
\hline H. exoprolifera & $\begin{array}{l}\text { Mangrove leaf litter } \\
\text { Rhizophora mangle }\end{array}$ & Bahamas, Japan, Philippines & Ho et al. 1992 \\
\hline H. polymorphica & Eucalyptus sp. & Australia, Taiwan & $\begin{array}{l}\text { Gerrettson-Cornell and } \\
\text { Simpson 1984, Pang et al. } \\
2014\end{array}$ \\
\hline H. porrigovesica & $\begin{array}{l}\text { Mangrove leaf litter } \\
\text { Sonneratia alba }\end{array}$ & Japan, Philippines & Nakagiri et al. 2001 \\
\hline H. vesicula & $\begin{array}{l}\text { Prunus laurocerasus } \\
\text { (in seawater) } \\
\text { Avicennia, Rhizophora, } \\
\text { Sonneratia, Xylocarpus }\end{array}$ & $\begin{array}{l}\text { Canada, Japan, Philippines, } \\
\text { Taiwan }\end{array}$ & $\begin{array}{l}\text { Anastasiou and Churchland } \\
1969 \\
\text { Nakagiri 2000, } \\
\text { Leaño 2001, Pang et al. 2014, } \\
\text { Caguimbal et al. } 2019\end{array}$ \\
\hline \multicolumn{4}{|l|}{ Phytopythium } \\
\hline Pp. dogmae & Mangrove leaf litter & Philippines & Bennett et al. 2017b \\
\hline Pp. kandeliae & $\begin{array}{l}\text { Avicennia lanata } \\
\text { Rhizophora apiculata } \\
\text { Sonneratia sp. } \\
\text { Kandelia candel }\end{array}$ & Brazil, Japan, Philippines, Taiwan & $\begin{array}{l}\text { Nakagiri 2000, Leaño 2001, } \\
\text { Marano et al. 2014, Ho et al. } \\
1991\end{array}$ \\
\hline Pp. leanoi & Mangrove leaf litter & Philippines & Bennett et al. 2017b \\
\hline \multicolumn{4}{|l|}{ Calycofera } \\
\hline C. cryptica & Avicennia sp. & Belize & Bennett et al. 2017c \\
\hline C. operculata & Avicennia marina & Australia & $\begin{array}{l}\text { Pegg and Alcorn } 1982 \\
\text { Bennett et al. 2017c }\end{array}$ \\
\hline
\end{tabular}


Table 2 (cont.). Summary of mangrove or saltmarsh oomycetes

\begin{tabular}{|c|c|c|c|}
\hline & Substrate & Country & Reference \\
\hline \multicolumn{4}{|l|}{ Salisapiliaceae } \\
\hline \multicolumn{4}{|l|}{ Salisapilia } \\
\hline S. bahamensis & Mangrove leaf litter & Bahamas, Philippines & Bennett and Thines 2019 \\
\hline S. coffeyi & Rhizophora mangle & Bahamas & Bennett and Thines 2019 \\
\hline S. elongata & Mangrove leaf litter & Philippines, Taiwan & Bennett and Thines 2019 \\
\hline \multirow[t]{2}{*}{ S. epistomia } & $\begin{array}{l}\text { Rhizophora, Sonneratia, } \\
\text { Xylocarpus } \\
\text { Decaying leaf, Rhizophora }\end{array}$ & Japan, Philippines, USA & $\begin{array}{l}\text { Nakagiri 2000, Leaño 2001, } \\
\text { Bennett and Thines } 2019\end{array}$ \\
\hline & & & Fell and Master 1975 \\
\hline S. nakagirii & Spartina alterniflora & USA & Hulvey et al. 2010 \\
\hline S. masteri & Avicennia germinans & Bahamas & $\begin{array}{l}\text { Nakagiri et al. 1994, } \\
\text { Bennett and Thines } 2019\end{array}$ \\
\hline S. mycoparasitica & Rhizophora & Japan, Malaysia & $\begin{array}{l}\text { Fell and Master 1975, } \\
\text { Nakagiri 2000, Bennett and } \\
\text { Thines } 2019\end{array}$ \\
\hline S. sapeloensis & Spartina alterniflora & USA & Hulvey et al. 2010 \\
\hline S. tartarea & Spartina alterniflora & USA & $\begin{array}{l}\text { Nakagiri et al. 1994, } \\
\text { Hulvey et al. 2010, } \\
\text { Bennett and Thines } 2019\end{array}$ \\
\hline \multicolumn{4}{|l|}{ Salispinaceae } \\
\hline \multicolumn{4}{|l|}{ Salispina } \\
\hline S. hoi & Mangrove leaf litter & Philippines & Bennett et al. 2018b \\
\hline S. intermedia & $\begin{array}{l}\text { Rhizophora mangle, } \\
\text { Laguncularia racemosa }\end{array}$ & Brazil & Li et al. 2016 \\
\hline S. lobata & $\begin{array}{l}\text { Xylocarpus } \\
\text { Rhizophora }\end{array}$ & $\begin{array}{l}\text { Japan, Malaysia, Philippines, Sey- } \\
\text { chelles, Singapore, Taiwan, Thai- } \\
\text { land, USA, Vietnam }\end{array}$ & $\begin{array}{l}\text { Fell and Master 1975, Nakagiri } \\
\text { 2000, Leaño 2001, Li et al. } \\
2016 \text {, }\end{array}$ \\
\hline S. spinosa & Rhizophora & $\begin{array}{l}\text { Bahamas, Colombia, Grand Cayman, } \\
\text { Haiti, Japan, Philippines, Thailand, } \\
\text { Taiwan, The Netherlands, Trinidad } \\
\text { and Tobago }\end{array}$ & $\begin{array}{l}\text { Fell and Master 1975, Pang et } \\
\text { al. 2014, Li et al. } 2016, \\
\text { Caguimbal et al. } 2019\end{array}$ \\
\hline
\end{tabular}


oomycete families: Peronosporaceae (Calycofera, Halophytophthora, Phytophthora, and Phytopythium), Salispinaceae (Salispina), and Salisapiliaceae (Salisapilia). Thus, the genus concept of Halophytophthora encompassing all Phytophthora-like species in estuarine environments is obsolete since several groups of saprotrophic oomycetes are found in the mangrove environment (i.e. Calycofera, Phytophthora, Phytopythium, Salisapilia, and Salispina) (see Lara and Belbahri 2011, Thines 2014, Nigrelli and Thines 2013, Bennett et al. 2017a, 2017b, 2017c, 2018, 2019). In addition, the premise "mangrove or estuarine oomycetes" is arbitrary, as it does not represent any phylogenetic significance within the genera that harbor such species, as several genera include species from both freshwater and marine habitats (Fig. 2).

Several taxa previously classified as Halophytophthora were transferred to Salisapilia by Bennett and Thines (2019), which now contains 9 species and can be considered as a rather species-rich genus. Further, the phylogenetic and morphological distinctiveness of the species justifies the recognition of Salisapiliaceae as a family independent from Pythiaceae and Peronosporaceae (Fig. 2).

The genus Salispina was previously considered as an incertae sedis taxon but was resolved based on combined morphological and phylogenetic analyses (Bennett et al. 2018). This genus is now accommodated into a family of its own, Salispinaceae, and is phylogenetically related to Rhipidiaceae. These two families are both placed in the Rhipidiales - a group capable of growing in an environment with reduced oxygen concentration. A fourth congener was introduced for Salispina, $S$. hoi which is the third species of the genus reported for the Philippines (Bennett et al. 2018).

Apart from the three genera mentioned above, the genus Phytopythium seems to be another frequent inhabitant of mangroves (Lara and Belbahri 2011, Thines 2014). From the Philippines, $P p$. leanoi, a sister taxon to $P p$. kandeliae and $P p$. dogmae has recently been described. Phytopythium leanoi is morphologically divergent from $P p$. kandeliae based on several combined characters while $P p$. dogmae, is considered a member of the clade 2 group of the genus Phytopythium (Bennett et al. 2017c).

The sister genus to Phytopythium is Calycofera. The latter genus was described to preserve the well-delineated morphological diagnosis of Phytopythium. Apart from the type species, Calycofera operculata ( $\equiv$ Halophytophthora operculata), a second congener, C. cryptica, was described based on nucleotide sequence divergence (Bennett et al. 2017a)

Despite the recent efforts outlined above, there are still taxa that remain enigmatic and require further analysis. An example is the genus Phytophthora which is an assemblage of species that is paraphyletic or polyphyletic with respect to the downy mildews and grouped into approximately 8 to 10 clades without clear-cut synapomorphies (Runge et al. 2011). Phytophthora harbors mangrove oomycetes (Bennett et al. 2017b, Bennett and Thines 2017) (i.e. in clades 9/10, 6 and 2) but it is unclear if these species act as opportunistic plant pathogens of mangroves or are purely saprotrophic in this environment. Also the Pythiaceae remains problematic despite the proposed changes of Uzuhashi et al. (2010) to resolve the polyphyly of Pythium by describing the genera Elongisporangium, Globisporangium, Pilasporangium and Ovatisporangium (as a later synonym of Phytopythium, Bala et al. 2010), apart from Pythium in a strict sense. Unfortunately, the phylogeny presented by Uzuhashi et al. (2010) was not wellresolved and left some mangrove oomycetes with unclear phylogenetic affinity.

After the revisions outlined above, there are only five congeners of Halophytophthora left $(H$. avicenniae, $H$. batemanensis, $H$. polymorphica, $H$. vesicula, and the freshwater isolate $H$. fluviatilis). In addition, two species, Halophytophthora exoprolifera and $H$. porrigovesica, are not clearly nested within Halophytophthora s.str. clade but might either represent a sister taxon to Halophytophthora or belong to Nothophytophthora (Jung et al. 2017), or the paraphyletic genus Phytophthora. Since there is no clear morphological or phylogenetic support for $H$. exoprolifera as a new genus of its own, it has so far been retained in Halophtophthora. Similarly, H. porrigovesica was not officially transferred to Phytophthora, since the latter taxon itself is unresolved and the morphology of H. porrigovesica does not fit well to either Nothophytophthora or clades 9 and 10 of Phytophthora.

Beakes and Thines (2017) emphasized the importance of saprotrophic Peronosporomycetes (including mangrove and saltmarsh oomycetes) for better understanding the evolutionary patterns of oomycetes in general. This paraphyletic group is phylogenetically placed between the downy mildews and Saprolegniomycetes and is key for understanding ecological shifts (i.e. habitat change), lifestyle transitions (e.g. biotrophy, saprotrophy), and thallus organization in oomycetes. Therefore, more research into these interesting species is likely to reveal more insights into oomycete evolution, diversity, and ecological functions in the years to come.

\section{Literature Cited}

Anastasiou C.J. \& L.M. Churchland, 1969. Fungi on decaying leaves in marine habitats. Canadian Journal of Botany, 47: 251-257.

Antazo, I.M., 2014. Marine straminipilous organisms isolated 
from decaying leaves of Rhizophora spp. from Manila Marina Baytown access road and Boac, Marinduque Province. Cognoscere: SPUQC Student Research Journal, 9: $1-16$.

Bala K., G.P. Robideau, C.A. Lévesque, A.W.A.M. de Cock, Z.G. Abad, A.M. Lodhi, S. Shahzad, A. Ghaffar \& M.D. Coffey, 2010. Phytopythium Abad, de Cock, Bala, Robideau, Lodhi and Lévesque, gen. nov. and Phytopythium sindhum Lodhi, Shahzad and Lévesque, sp. nov. Persoonia, 24: 136-137.

Beakes G. \& M. Thines, 2017. Hyphochytriomycota and Oomycota. In: Archibald, J.M., A.G.B. Simpson \& C.H. Slamovits, (ed.), Handbook of the Protists, $2^{\text {nd }}$ ed. Springer International Publishing, Cham, Switzerland. pp. 435-505.

Bennett R.M., A.W.A.M. de Cock, C.A. Lévesque \& M. Thines, 2017a. Calycofera gen. nov., an estuarine sister taxon to Phytopythium (Peronosporaceae). Mycological Progress, 16: 947-954.

Bennett R.M., G.R. Dedeles \& M. Thines, 2017b. Phytophthora elongata (Peronosporaceae) is present as an estuarine species in Philippine mangroves. Mycosphere, 8: 959-967.

Bennett R.M., M.K. Devandera, G.R. Dedeles \& M. Thines, 2018. A revision of Salispina, its placement in a new family, Salispinaceae (Rhipidiales), and description of a fourth species, S. hoi sp. nov. IMA Fungus, 9: 259-269.

Bennett R.M., B. Nam, G.R. Dedeles \& M. Thines, 2017c. Phytopythium leanoi sp. nov. and Phytopythium dogmae sp. nov., Phytopythium species associated with mangrove leaf litters from the Philippines. Acta Mycologica, 52(2): 1103. https://doi. org/10.5586/am.1103

Bennett R.M. \& M. Thines, 2017. Confirmation that Phytophthora insolita (Peronosporaceae) is present as a marine saprotroph on mangrove leaves and first report of the species for the Philippines. Nova Hedwigia, 105: 185196.

Bennett R.M. \& M. Thines, 2019. Revisiting Salisapiliaceae. Fungal Systematics and Evolution, 3: 171-184.

Borines L.M., V.G. Palermo, G.A. Guadalquiver, C. Dwyer, A. Drenth, R. Daniel \& D.I. Guest, 2014. Jackfruit decline caused by Phytophthora palmivora (Butler). Australasian Plant Pathology, 43: 123-129.

Caguimbal N.A.L.E., M.K.P. Devanadera, R.M. Bennett. K.H.V. Arafiles, K. Watanabe, T. Aki \& G.R. Dedeles, 2019. Growth and fatty acid profiles of Halophytophthora vesicula and Salispina spinosa from Philippine mangrove leaves. Letters in Applied Microbiology, 69: 221-228.

Celino, M.S., 1933. Blight of cinchona seedlings. Philippine Agriculture, 23: 111-123.

Clara, F.M. 1928. A Phytophthora disease of santol seedlings.
Philippine Journal of Science, 35: 411-425.

De Cock, A.W.A.M., A.M. Lodhi, T.L. Rintoul, K. Bala, G.P. Robideau, Z.G. Abad, M.D. Coffey, S. Shahzad \& C.A. Lévesque, 2015. Phytopythium: molecular phylogeny and systematics. Persoonia, 34: 25-39.

Del Rosario, M.S., 1968. A handbook of citrus diseases in the Philippines. UPCA Technical Bulletin, 31: 1-33.

Dogma, I.J., 1975. Of Philippine mycology and lower fungi. Kalikasan, Philippine Journal of Biology, 4: 69-105.

Dogma, I.J., 1986. Zoosporic Fungi. In: Guide to Philippine Flora and Fauna, vol. 1. Natural Resources Management Center, Ministry of Natural Resources and University of the Philippines. Quezon City. pp. 1-199.

Ela, V.M., 1968. Notes on diseases of orchids in the Philippines. Philippine Agriculture, 4: 531-537.

Fell, J.W. \& I.M. Master, 1975. Phycomycetes (Phytophthora spp. nov. and Pythium sp. nov.) associated with degrading mangrove (Rhizophora mangle) leaves. Canadian Journal of Botany, 53: 2908-2922.

Fell, J.W. \& I.M. Master, 1980. The association and potential role of fungi in mangrove detrital systems. Botanica Marina, 23: 257-263.

Gerrettson-Cornell L. \& J. Simpson, 1984. Three new marine Phytophthora species from New South Wales. Mycotaxon, 19: 453-470.

Hatai K., Z.B. Bian, M.C.L. Baticados \& S. Egusa, 1980. Studies on fungal diseases in crustaceans: II. Haliphthoros philippinensis sp. nov. isolated from cultivated larvae of the jumbo tiger prawn (Penaeus monodon). Transactions of the Mycological Society of Japan, 21: 47-55.

Ho H.H., H.S. Chang \& S.Y. Hsieh, 1991. Halophytophthora kandeliae, a new marine fungus from Taiwan. Mycologia, 83: 419-424.

Ho H.H., H.S. Chang \& S.H. Huang, 2003. Halophytophthora elongata, a new marine species from Taiwan. Mycotaxon, 85: 417-422.

Ho H.H. \& S.C. Jong, 1990. Halophytophthora, gen. nov., a new member of the family Pythiaceae. Mycotaxon, 36: $377-$ 382.

Ho H.H., A. Nakagiri \& S.Y. Newell, 1992. A new species of Halophytophthora from Atlantic and Pacific subtropical islands. Mycologia, 84: 548-554.

Hulvey J., S. Telle, L. Nigrelli, K. Lamour \& M. Thines, 2010. Salisapiliaceae - a new family of oomycetes from marsh grass litter of southeastern North America. Persoonia, 25: 109-116.

Jung T., B. Scanu, J. Bakonyi, D. Seress, G.M. Kovács, A. Durán, et al., 2017. Nothophytophthora gen. nov., a new sister genus of Phytophthora from natural and semi-natural 
ecosystems. Persoonia, 39: 143-174.

Lara E. \& L. Belbahri, 2011. SSU rRNA reveals major trends in oomycete evolution. Fungal Diversity, 49: 93-100.

Leaño, E.M., 2001. Straminipilous organisms from fallen mangrove leaves from Panay Island, Philippines. Fungal Diversity, 6: 75-81.

Leaño, E.M., 2002. Haliphthoros spp. from spawned eggs of captive mud crab, Scylla serrata, broodstrocks. Fungal Diversity, 9: 93-103.

Leaño E.M., E.B.G. Jones \& L.L.P. Vrijmoed, 2000. Why are Halophytophthora species well adapted to mangrove habitats? Fungal Diversity, 5: 131-151.

Lee, A.H., 1921. Observations on previously unreported or noteworthy plant diseases in the Philippines. Philippine Agricultural Review, 14: 422-434.

Leong, W.F., T.K. Tan \& E.B.G. Jones, 1998. Lignicolous marine fungi of Singapore. Canadian Journal of Botany, 66: 2167-2170.

Lévesque C.A. \& A.W.A.M. de Cock, 2004. Molecular phylogeny and taxonomy of the genus Pythium. Mycological Research, 108: 1363-1383.

Li G.J., K.D. Hyde, R.L. Zhao, S. Hongsana, F.A. Abdel-Aziz, et al., 2016. Fungal diversity notes 253-366: taxonomic and phylogenetic contributions to fungal taxa. Fungal Diversity, 78: $1-237$.

Man in'T Veld, W.A., K.C.H.M. Rosendahl, H. Brouwe \& A.W.A.M. de Cock, 2011. Phytophthora gemini sp. nov., a new species isolated from the halophilic plant Zostera marina in the Netherlands. Fungal Biology, 115: 724-732.

Marano A.V., A.L. Jesus, J.I. de Souza, E.M. Leaño, T.Y. James, G.H. Jeronimo, A.W.A.M. de Cock \& C.L.A. PiresZottarelli, 2014. A new combination in Phytopythium: P. kandeliae (Oomycetes, Straminipila). Mycosphere, 5: 510522.

Mendiola, N. \& R.B. Espino, 1916. Some phycomycetous diseases of cultivated plants in the Philippines. Philippine Agriculturist and Forester, 5: 65-72.

Nakagiri, A., 2000. Ecology and biodiversity of Halophytophthora species. Fungal Diversity, 5: 153-164.

Nakagiri, A., 2002. Diversity and phylogeny of Halophytophthora (Oomycetes). In: Book of abstracts of the IMC7 - The $7^{\text {th }}$ International Mycological Congress. Oslo, 11-17 August 2002. 55: 19

Nakagiri, A., T. Ito, L. Manoch \& M. Tanticharoen, 2001. A new Halophytophthora species, H. porrigovesica, from subtropical and tropical mangroves. Mycoscience, 42: 3341.

Nakagiri, A., S.Y. Newell \& T. Ito, 1994. Two new Halophytophthora species, H. tartarea and H. masteri, from intertidal decomposing leaves in saltmarsh and mangrove regions. Mycoscience, 35: 223-232.

Nakagiri, A., S. Tokumasu, H. Araki, S. Koreeda \& K. Tubaki, 1989. Succession of fungi in decomposing mangrove leaves in Japan. In: Hattori T., Y. Ishida, Y. Maruyama, R. Morita \& A. Uchida, (ed.), Recent Advances in Microbial Ecology (Proceedings of the $5^{\text {th }}$ International Symposium on Microbial Ecology. Japan Science Society Press, Tokyo. Pp. 297-301.

Newell, S.Y., J.D. Miller \& J.W. Fell, 1987. Rapid and pervasive occupation of fallen mangrove leaves by a marine zoosporic fungus. Applied and Environmental Microbiology, 53: 2464-2469.

Nigrelli, L. \& M. Thines, M., 2013. Tropical oomycetes in the German Bight - Climate warming or overlooked diversity. Fungal Ecology, 6: 152-160.

Ocfemia, G.O., 1925. The Phytophthora disease of eggplant in the Philippine Islands. Philippine Agriculture, 14: 317-328.

Pang, K.L., H.J. Lin, H.Y. Lin, Y.F. Huang \& Y.M. Chen, 2014. Production of arachidonic and eicosapentaenoic acids by the marine oomycete Halophytophthora. Marine Biotechnology, 17: 121-129.

Pegg, K.G. \& J.L. Alcorn, 1982. Phytophthora operculata sp. nov., a new marine fungus. Mycotaxon, 16: 99-102.

Pegg, K.G. \& J.L. Foresberg, 1981. Phytophthora in Queensland mangroves. Wetlands, 1: 2-3.

Primavera, J., 2000. Development and conservation of Philippine mangroves: Institutional issues. Ecological Economics, 35: 91-106.

Quebral, F.C., A.N. Pordesimo, T.T. Reyes \& B.P. Tamayo, 1962. Heart rot of pineapple in the Philippines. Philippine Agriculture, 46: 432-450.

Quimio, T.H. \& A.J. Quimio, 1974. Compendium of postharvest and common diseases of fruits in the Philippines. UPCA Technical Bulletin 34.

Raghukumar, S., S. Sharma, C. Raghukumar, V. Sathe-Pathak \& D. Chandramohan, 1994. Thraustochytrid and fungal component of marine detritus. IV. Laboratory studies on decomposition of leaves of the mangrove Rhizophora apiculata Blume. Journal of Experimental Marine Biology and Ecology, 183: 113-131.

Reinking, O.A., 1919. Phytophthora faberi Maubl: the cause of coconut bud rot in the Philippines. Philippine Journal of Science, 14: 131-150.

Runge, F., S. Telle, S. Ploch, E. Savory, B. Day, R. Sharma \& M. Thines, 2011. The inclusion of downy mildews in a multi-locus dataset and its reanalysis reveals a high degree of paraphyly in Phytophthora. IMA Fungus, 2: 163-171.

Say, E.K., A.T.V. Yabut, N.E.T. Cinco, N.A.L.E. Caguimbal, 
M.K.P. Devanadera, R.M. Bennett, et al., 2017. Growth and fatty acid production of Halophytophthora S13005YL1-3.1 under different salinity and $\mathrm{pH}$ levels. Philippine Agricultural Scientist, 100: 6-11.

Tan, T.K. \& W.F. Leong, 1992. Lignicolous fungi of tropical mangrove wood. Mycological Research, 96: 413-414.

Tan, T.K. \& C.L. Pek, 1997. Tropical mangrove leaf litter fungi in Singapore with emphasis on Halophytophthora. Mycological Research, 2: 165-168.

Teodoro, N.G., 1926. Rubber tree diseases and their control. Philippine Agriculture Review, 19: 63-73.

Thines, M. 2014. Phylogeny and evolution of plant pathogenic oomycetes - a global overview. European Journal of Plant Pathology, 138: 431-447.

Tsao P.H., L.C. Gruber, L.A. Portales, A.M. Gochangco, P.B. Luzaran, A.B. de los Santos \& H. Pag, 1994. Some new records of Phytophthora crown and root rots in the Philippines and in world literature. Phytopathology, 84: 871. [Abstract]

van der Plaats-Niterink, A.J., 1981. Monograph of the genus Pythium. Studies in Mycology, 21: 1-242.

Weston, W.H., 1920. Philippine down mildew of maize. Journal of Agricultural Research, 19: 97-122.

Wijayawardene, N.N., K.D. Hyde, L.K.T. Al-Ani, L. Tedersoo, D. Haelewaters, K.C. Rajeshkumar, et al., 2020. Outline of Fungi and fungus-like taxa. Mycosphere, 11: 1060 - 1456.

Yang, X. \& C. Hong. 2014. Halophytophthora fluviatilis sp. nov. from freshwater in Virginia. FEMS Microbiology Letters, 352: 230-237. 